\title{
Study on Hydraulic Characteristic of the Tube Settler
}

\author{
Yonghai $\mathrm{Yu}^{*}$, Dong Liu and Xiaofeng Cui \\ College of Water Conservancy and Hydropower Engineering, Hohai University, Nanjing 210098, China \\ ${ }^{*}$ Corresponding author
}

\begin{abstract}
Little information could be found about formulas and coefficients for calculating the head loss of tube settler, which makes it difficult for the designers to get the values. Herein, using computational fluid dynamics software FLUENT as the platform, the hydraulic characteristics involving in water flow regime, velocity distribution and pressure distribution of flow were studied via the standard $k-\varepsilon$ turbulence model with the SIMPLEC algorithm and numerical simulation on the flow in tube settler based on the assumptions that the free water surface remains flat as a stress-free plane of symmetry and the velocity distribution and free outflow are prescribed on inlet and outlet boundaries respectively. The head loss of the tube settler was obtained from the numerical simulation, which was in good agreement with the measurement for actual engineering. The method based on numerical simulation is a new and good approach to determine the head loss of tube settler.
\end{abstract}

Keywords-tube settler; numerical simulation; head loss; turbulence model

\section{INTRODUCTION}

Inclined settling tank is designed according to the shallow pool theory proposed by Hazen in the beginning of twentieth Century[1].Inclined settling tank can be used to reduce the area of the settling pond or increase the processing capacity, and also can improve the hydraulic conditions,so that the surface load can be improved effectively.In the direction of the relative flow and granular flow, the inclined settling tanks commonly used in engineering are usually divided into same direction flow type, anisotropic flow type and horizontal flow type. Most of the current inclined settling tank are tube settler.

There are two modes of watering into inclined settling tank, pumping and artesian flow. For artesian flow mode, due to the settling tank built in the bed of the river, the head loss will be required as small as possible to reduce the water level of inlet river and the height of the wall of settling tank. So it is necessary to determine the head loss accurately in the design of settling tank. When calculating the head loss of settling tank with the help of existing design manual, the coefficient of head loss will be difficult to determine for some special arrangement, such as the perforated wall between settling tank inlet passage and distribution zone. Designing with empirical valuation will appear big error. Numerical simulation method will be used to determine the head loss of settling tank in the paper.

Currently, there are some research results about numerical simulation of setting tank. He Guo-jian[2] established a model to simulate process of suspended solids in flat flow settling tank, using FLUENT software $\mathrm{k}-\varepsilon$ turbulence model to simulate the water and suspended solids movement of settling tank. Cao Yue-bo[3]set up a three-dimensional two-phase flow model based on the high Reynolds number $\mathrm{k}-\varepsilon$ turbulence model, which verified the reliability of the numerical simulation. On the basis of vertical flow settling tank, Zhang Hong-yuan[4]added inclined plate to form a modified inclined plate settling tank, and the optimization of the structure parameters of the improved inclined plate sedimentation tank was determined by the FLUENT commercial software. It is feasible to use the FLUENT software to simulate the settling tank. The FLUENT software is used as the numerical simulation platform, and the feasibility of the numerical simulation is verified by the simulation results.

\section{INTRODUCTION TO THE PROJECT}

Figure 1 is the principle diagram of tube settler in Jiangsu province, China. Muddy water flows into the inlet pipe and enters the inlet passage and then flows through the wall holes (shown in Figure 2) to the distribution zone and then clean water from inclined pipes outflows to the outlet river.

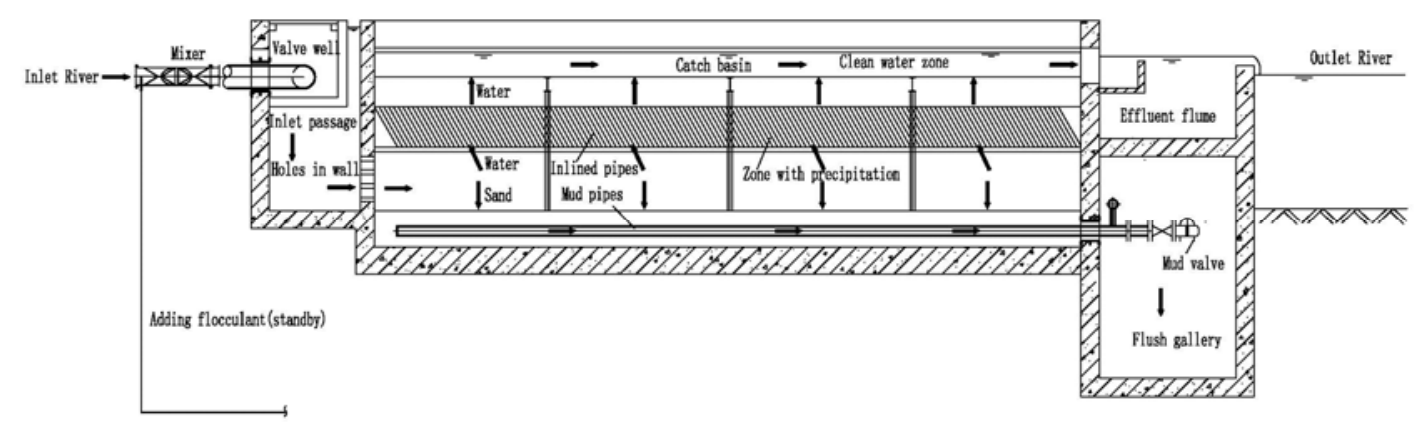

FIGURE I. PRINCIPLE DIAGRAM OF THE TUBE SETTLER 
The settling tank with muddy water flowing into all the year round is located in the upstream of the river. The total treating capacity of the settling tank with four same separated pools is $8 \times 10^{4} \mathrm{~m}^{3} / \mathrm{d}$. Water level in the outlet of settling tank depends on the water landscape of river in the city and adding the head loss of settling tank, the water level of the inlet river will be obtained. If the head loss from the calculation is too large, the water level of the inlet river will be higher and the elevation of the center line of the inlet pipe may be confirmed higher, which will bring disadvantageous effect of life and industrial production.

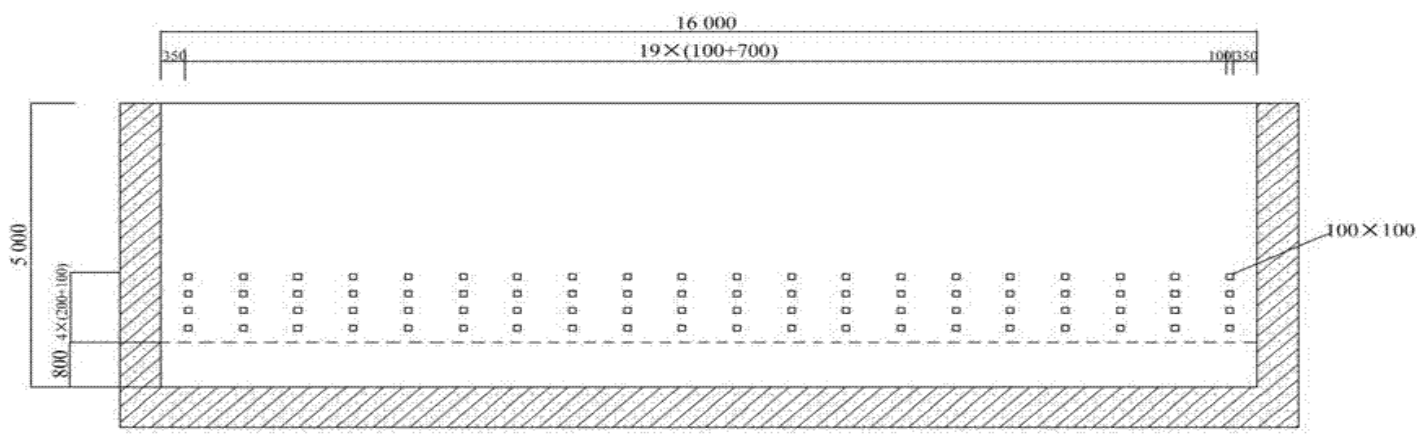

FIGURE II. LAYOUT OF THE HOLES IN THE WALL(UNIT:MM)

\section{MATHEMATICAL MODEL AND BOUNDARY CONDITIONS}

\section{A. Geometry Size and Gambit Modeling of Settling Tank}

Because of the low velocity and small Reynolds number, the flow is mostly laminar in inclined pipe zone and the head loss is very small. So space of inclined tubes [5] was not simulated specifically and the space of inclined tube was set to 0.1 meter. Although the real effect of precipitation cannot be reflected, the effect of the flow direction of motion can be reflected.

The model of settling tank will be meshed with structured and unstructured mesh (Figure 3 ) after completing 3D model in Gambit interface. The independence of mesh should be examined. When the results from calculation with FLUENT no longer change with the changed mesh density, the mesh independence will be confirmed. The final mesh number is 1015116.

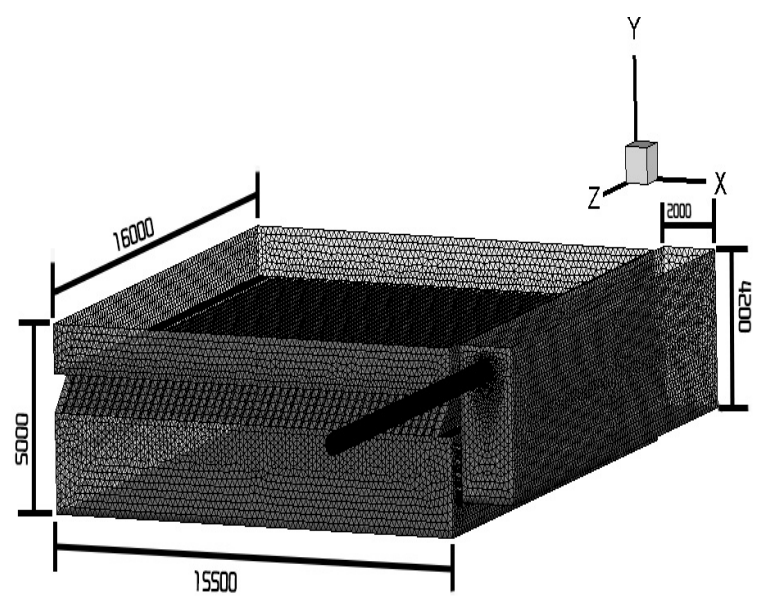

FIGURE III. MESH GENERATION OF THE TUBE SETTLER(UNIT:MM)

\section{B. Calculation Method and Boundary Conditions}

The standard k-eturbulence model and second-order upwind scheme and SIMPLEC algorithm are selected. The residuals of the convergent parameters, such as continuity, velocity, $\mathrm{k}, \varepsilon$, are set to 0.0001 .

When the concentration of suspended particles is low, the impact of solids to liquid is very small. The head loss of settling tank will be focused on studying, the solid particles can be neglected[6]. Adopting single-phase flow model to analysis the flow field of settling tank will save the calculation time.

The boundary conditions are listed below:

1) Inlet boundary: Selecting the velocity-inlet and assuming that the uniform distribution of velocity. The treating of single pool is $2 \times 10^{4} \mathrm{~m}^{3} / \mathrm{d}$ and the diameter of inlet pipe is $500 \mathrm{~mm}$ and then the inlet velocity is $1.18 \mathrm{~m} / \mathrm{s}$. Meanwhile, the pipeline length is extended to 20 times the diameter of the pipe, so that the flow velocity distribution can keep consistent with the actual velocity.

2) Outlet boundary: selecting outflow.

3) Solid wall boundary condition: no slip boundary conditions.

4) Free surface boundary: when calculating the water flow, the free surface is assumed by the rigid cover.

\section{SOLVING RESUlts}

\section{A. Flow Pattern and Velocity Distribution}

The head loss of tube settler is closely related with the state of the flow. When fluid flows through partial obstruction, such as fluid flows through the inlet pipe to inlet passage and passes through the wall holes into the water distribution zone, the rapid change of local boundary will lead to change of flow state and the velocity distribution and creating a vortex region will result in head loss. So it is very important to analyze the flow state and flow velocity of the settling tank. 
The coordinate origin of the model is in the center of the inlet passage. In order to analyze the flow state, respectively cutting along $\mathrm{x}=0 \mathrm{~m}, \mathrm{y}=-1.55 \mathrm{~m}, \mathrm{z}=4.4 \mathrm{~m}$ can obtain three sections. Figure 4, Figure 5 and Figure 6 are the velocity vector diagram in three sections.

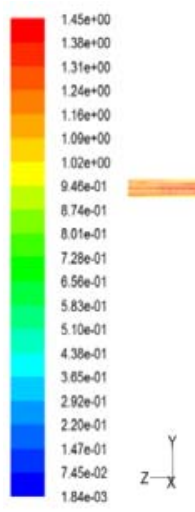

FIGURE IV. THE VELOCITY VECTOR OF INLET CROSS SECTION

It can be seen from the velocity vector diagram of the inlet section of Figure 4, outlet velocity of the inlet pipe is very large and then impacts with fluid of low flow velocity in the inlet corridor, which generates collision loss. In addition, submerged depth of the inlet pipe is not deep and form a jet flow in the outlet, which mixed with the static fluid because of turbulent fluctuation when the jet flow enter into the corridor. Surrounding fluid is entrained by jet fluid, which caused entrainment phenomenon and formed a large vortex that made the flow state unsmooth and increased the energy loss.

After water flows into the water distribution zone, if the turbulence is very intensity, the distance of sediment transport is large in the pool, which will not be conducive to the settlement of sediment. So wall holes between the inlet corridor and water distribution zone should be set, which can adjust the water flow state and make velocity distribution more uniform with conduciving to the settlement of sediment.As shown in Figure 6 and Figure 5, entrance velocity reaches $1.18 \mathrm{~m} / \mathrm{s}$ and the velocity of the wall hole is about $0.3 \mathrm{~m} / \mathrm{s}$ in the settling tank, then water flow rate in the water distribution zone is reduced to about $0.04 \mathrm{~m} / \mathrm{s}$ through the wall holes, which will be more conducive to the settlement.

From the velocity vector diagram can be seen, flow passed through the wall holes and entered into the water distribution zone with larger speed. Fluid particles colliding and changed velocity distribution can result in energy loss in fluid acceleration and deceleration process.Meanwhile, although the rate of the water distribution area is very low, from the velocity vector diagram can be seen that there is many vortices region with almost zero velocity. Vortexes mainly exist the outlet of the wall hole, central section of the settling tank body and result in head loss, which will cause adverse influence of the effect of precipitation.

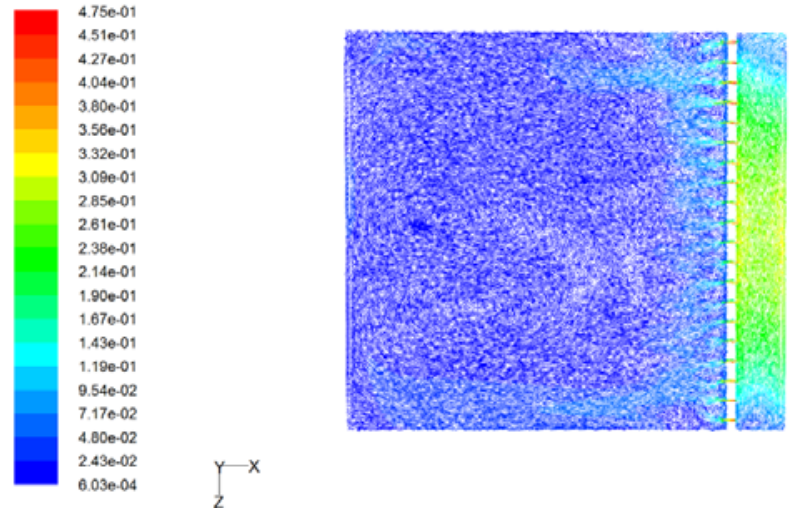

FIGURE V. THE VELOCITY VECTOR OF THE CROSS SECTION WITH Y DIRECTION

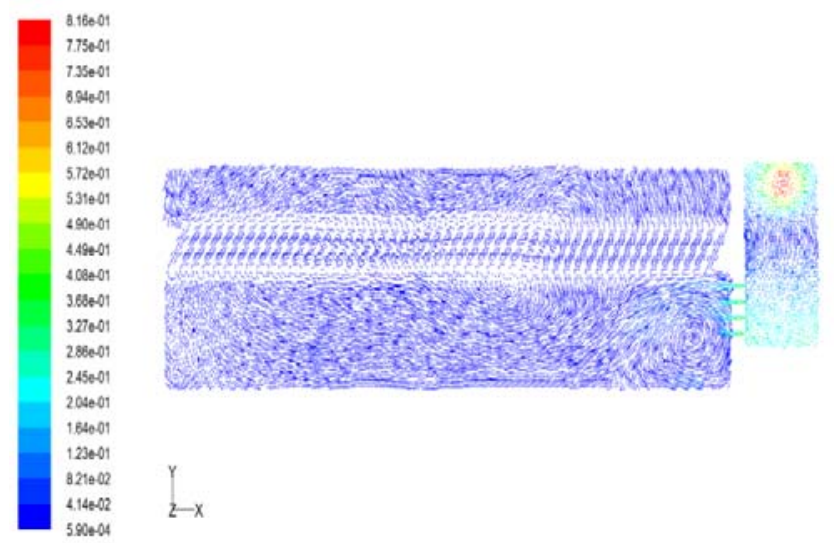

FIGURE VI. THE VELOCITY VECTOR OF THE CROSS SECTION WITH Z DIRECTION

\section{B. Calculation of Head Loss}

Total pressure, static pressure and pressure are three concepts in FLUENT and indicated as $p_{0}, p_{s}, p_{z}^{I}$ separately and their units are $\mathrm{Pa}$. Their relationship was defined according to below formula:

$$
\begin{aligned}
& p_{0}=p_{s}+\rho|v|^{2} \\
& p_{s}=p^{\prime}{ }_{s}+\rho_{0} g z
\end{aligned}
$$

In the formula, $\mathrm{z}$ is geometrical elevation of pressure measurement points, $\mathrm{v}$ is the flow velocity.

Static pressure has included the position head in FLUENT and the actual pressure is piezometric level. Figure 7 is total pressure diagram of settling tank. From the total pressure distribution chart can be seen that pressure distribution is more uniform in the sedimentary area. However, the pressure distribution in the inlet corridor is high on both sides and low in the middle, which is caused by the uneven velocity distribution.

In the actual measurement, the water levels of inlet corridor and clean water zone were measured respectively. The result of the minus of two water levels plus submerged water head loss of the water inlet pipe can get the head loss of settling tank between outlet section of inlet pipe and surface of clean water area. With the total pressures of inlet and outlet from simulation 
results, the head loss of the tube settler was calculated to $0.089 \mathrm{~m}$ with Bernoulli's equation which was close with the measured $0.08 \mathrm{~m}$.In order to verify the validity of the model, the flow was changed to half of the design flow to get the head loss of $0.0212 \mathrm{~m}$, which is also close to the actual measurement result of $0.02 \mathrm{~m}$.
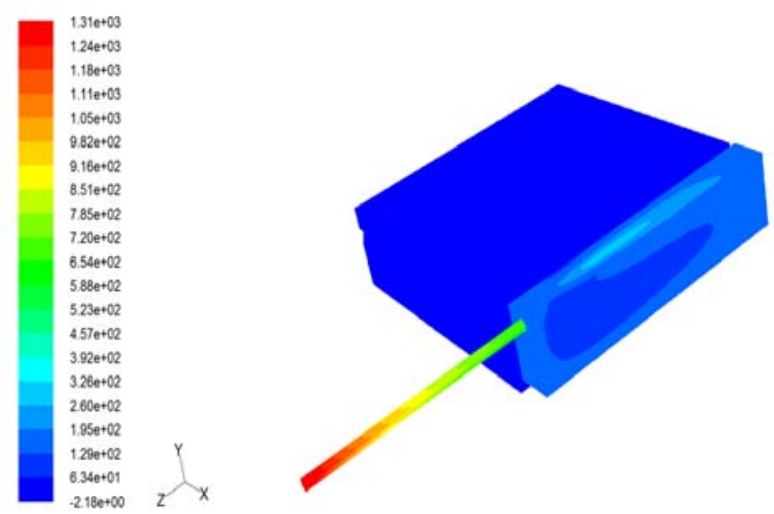

FIGURE VII. DISTRIBUTION OF TOTAL PRESSURE IN THE TUBE SETTLER

\section{CONCLUSIONS}

The tube settler in the river was studied in the paper. The flow state and velocity and pressure distribution can be obtained with numerical simulation of three-dimensional flow field and then can calculate the head loss of settling tank. Since the calculated values are consistent with the measured values, it is feasible to use the numerical simulation to determine the head loss of the tube settler. Analyzing the influence factors of the head loss of the pool, the water head loss of submerged flow accounted for larger proportion in the whole head loss of settling tank. So decreasing outlet velocity of inlet pipe can significantly reduce head loss of settling tank, which can provide a useful reference for the optimization of the hydraulic design of this type of settling tank.

\section{REFERENCES}

[1] Gao Tingyao, Gu Guowei, Water pollution control engineering, second ed., Higher Education Press,Beijing,2001.

[2] He Guojian, Wang Dewei, Simulation of suspended sediment deposition in rectangular settling tank.Journal of Tsinghua University,45(2005)1617-1620.

[3] Wang Xiaoling,Cao Yuebo,Zhang Mingxing, Three dimensional numerical simulation of solid-liquid two-phase flow in radial flow sedimentation tank,Engineering mechanics,26(2009)243-249.

[4] Zhang Hongyuan,Liquid-solid two phase flow simulation of improved tube settler and experiment research of separating property, Harbin industrial university,2011.

[5] Fang Xiaotao,Precipitation area flow simulation and design optimization research of high-density settlin tank in water treatment plant.Chongqing Jiaotong University,2010.

[6] Zhang Xiang, Theoretical analysis and numerical simulation of the double flow tube settler.Harbin Institute of Technology,2011.

[7] Wang Fujun.The principle and application of computational fluid dynamics software-CFD, Tsinghua University press, Beijing, 2004.

[8] B.E.Launder,D.B.Spalding,The Numerical Computation of Turbulent Flows. Computer Methods in Applied Mechanics and Engineering, 3(1974)269-289. 